\title{
How attention enhances spatial resolution: Evidence from selective adaptation to spatial frequency
}

\author{
MARISA CARRASCO, FANI LOULA, and YUN-XIAN HO \\ New York University, New York, New York
}

\begin{abstract}
In this study, we investigated how spatial resolution and covert attention affect performance in a texture segmentation task in which performance peaks at midperiphery and drops at peripheral and central retinal locations. The central impairment is called the central performance drop (CPD; Kehrer, 1989). It has been established that attending to the target location improves performance in the periphery where resolution is too low for the task, but impairs it at central locations where resolution is too high. This is called the central attention impairment (CAI; Yeshurun \& Carrasco, 1998, 2000). We employed a cuing procedure in conjunction with selective adaptation to explore (1) whether the $\mathrm{CPD}$ is due to the inhibition of low spatial frequency responses by high spatial frequency responses in central locations, and (2) whether the CAI is due to attention's shifting sensitivity to higher spatial frequencies. We found that adaptation to low spatial frequencies does not change performance in this texture segmentation task. However, adaptation to high spatial frequencies diminishes the CPD and eliminates the CAI. These results indicate that the CPD is primarily due to the dominance of high spatial frequency responses and that covert attention enhances spatial resolution by shifting sensitivity to higher spatial frequencies.
\end{abstract}

One of the most intriguing areas of current research in visual science concerns the role of attention in information processing. Visual attention allows us to select a certain location or aspect of a scene and grant it priority in processing. Spatial covert attention (hereafter referred to as attention) is the selective processing of visual information at a given location in the absence of eye movements to that location (Posner, 1980). Attention can be either voluntarily allocated to a given location according to goals - sustained attention - or involuntarily (reflexively) allocated in response to a cue that appears suddenly in the visual field - transient attention (Cheal \& Lyon, 1991; Jonides, 1981; Nakayama \& Mackeben, 1989).

Psychophysical studies using a variety of tasks indicate that attention affects early visual processing, including two basic dimensions of vision - contrast sensitivity (e.g., Cameron, Tai, \& Carrasco, 2002; Carrasco, PenpeciTalgar, \& Eckstein, 2000; Dosher \& Lu, 2000a, 2000b; Lee, Itti, Koch, \& Braun, 1999; Ling \& Carrasco, 2006; Lu \& Dosher, 1998, 2000) and spatial resolution. Support for enhanced spatial resolution is provided by studies on visual search (Carrasco \& McElree, 2001; Carrasco \& Yeshurun, 1998), acuity and hyperacuity (Carrasco, Williams, \& Yeshurun, 2002; Golla, Ignashchenkova, Haarmeier, \&

An NSF BCS-9910734 grant to M.C. supported this study. We thank all the members in the Carrasco lab, as well as Mike Landy, Maria-Luisa Martelli, Denis Pelli, and Yaffa Yeshurun for their comments on a draft of the manuscript. Correspondence and requests for materials should be addressed to M. Carrasco (e-mail: marisa.carrasco@nyu.edu).
Their, 2004; Shiu \& Pashler, 1995; Yeshurun \& Carrasco, 1999), and texture segmentation (Talgar \& Carrasco, 2002; Yeshurun \& Carrasco, 1998, 2000). Psychophysical studies on acuity (Yeshurun \& Carrasco, 1999) and texture segmentation (Yeshurun \& Carrasco, 1998, 2000) lend support and psychological reality to the neurophysiological studies showing that attention increases spatial resolution (Reynolds \& Desimone, 1999). Furthermore, other psychophysical studies suggest that attention allows a finer scale analysis. For instance, focusing attention on the target location reduces orientation thresholds through the operation of a smaller-scaled stimulus analyzer (Morgan, Ward, \& Castet, 1998). Likewise, an attended line is perceived as shorter because smaller attentional receptive fields mediate the processing of the attended stimulus (Tsal \& Shalev, 1996). Recently, it has been shown that transient attention also increases apparent spatial frequency and gap size (Gobell \& Carrasco, 2005).

The primary goal of this study was to directly test whether covert attention enhances spatial resolution by shifting sensitivity to higher spatial frequencies. Given that spatial resolution decreases with eccentricity, it follows that performance also decreases with eccentricity in most resolution tasks (see, e.g., Carrasco \& Frieder, 1997; Graham, 1989). Texture segmentation, in which an image must be parsed into two disparate regions of texture that differ along one or more dimensions, has typically been modeled as a process that occurs in parallel across retinal locations. It is not true, however, that all retinal locations are processed with the same efficiency. In fact, in a texture segmentation task in which the elements in the target and 
background differ in their orientation, performance peaks at midperipheral locations and drops as the target appears at more peripheral or central locations. This is referred to as the central performance drop, or CPD (Kehrer, 1989). This pattern of results has been replicated in a number of studies (e.g., Gurnsey, Di Lenardo, \& Potechin, 2004; Gurnsey, Pearson, \& Day, 1996; Morikawa, 2000; Talgar \& Carrasco, 2002; Yeshurun \& Carrasco, 1998, 2000), and it indicates that the texture segmentation task investigated here is not spatially invariant.

One interpretation of the CPD ascribes the pattern of performance to temporal factors. According to this view, the CPD can be explained by differences in the speed of visual processing as functions of eccentricity. Processing speed increases with distance from the fovea (Breitmeyer, 1984; Carrasco, McElree, Denisova, \& Giordano, 2003). Given that processing occurs at a slower rate in central vision, it is possible that the CPD may result from the interruption of processing in these locations by a mask presented shortly after the texture stimulus. In contrast, processing at midperipheral locations is faster and may be completed before presentation of the mask (Joffe \& Scialfa, 1995; Kehrer, 1989; Morikawa, 2000). However, given that the CPD can be obtained even without a backward mask (Potechin \& Gurnsey, 2003; Yeshurun \& Carrasco, 2000), the temporal explanation does not suffice.

The most widely accepted explanation for the CPD is based on spatial characteristics of the visual system; the impairment in performance reflects a mismatch between the scale of the texture and the dominant size of spatial filters, which for certain textures are too small in the fovea and too large in the periphery (Gurnsey et al., 1996; Kehrer \& Meinecke, 2003; Morikawa, 2000; Talgar \& Carrasco, 2002; Yeshurun \& Carrasco, 1998, 2000). Consistent with this explanation, performance with a larger texture scale peaks at farther eccentricities, reflecting the fact that the processing of this enlarged texture requires larger filters that are more abundant at farther eccentricities (Gurnsey et al., 1996; Kehrer, 1997; Yeshurun \& Carrasco, 1998).

Covert attention has been found to influence the shape of this function in a counterintuitive manner; attention does not benefit performance across all eccentricities. It improves performance where resolution is too low-that is, at peripheral locations-but hinders performance where resolution is already too high - that is, at central locations. We refer to this as the central attentional impairment (CAI). Peak performance is thus shifted toward farther eccentricities. Furthermore, the CAI changes as a function of texture scale. Performance is impaired in a larger range of central retinal locations as the scale of the texture increases, and the peak of the attentional function shifts to even farther eccentricities (Talgar \& Carrasco, 2002; Yeshurun \& Carrasco, 1998). The finding that attention improves or impairs performance on this texture segmentation task as a function of both target eccentricity and the scale of the texture is critical to the present study.

Psychophysical and neurophysiological studies indicate that the visual system extracts information by means of multiple "channels," or quasi-independent mechanisms whose bandwidths are about 1-2 octaves in spatial frequency and about $15^{\circ}$ in orientation (DeValois, Albrecht, \& Thorell, 1982; DeValois \& DeValois, 1988). Outputs from these individual filters, or channels, are responsible for spatial frequency and orientation tuning and appear to be processed simultaneously. They jointly determine detection and discrimination of spatial patterns via a normalization process; that is, the response of each individual filter is divided by the total response of a set of spatially overlapping filters responding to different spatial frequencies and orientations (Foley, 1994; Graham \& Sutter, 2000; Heeger, 1992). In general, receptive field size increases with eccentricity; thus, the peak frequency to which the channels respond decreases - the smaller the receptive fields, the higher the frequencies they encode (DeValois et al., 1982; DeValois \& DeValois, 1988; Morikawa, 2000).

The secondary goal of this study was to test the hypothesis that interfrequency (or cross-frequency) inhibition is a critical process underlying the CPD. Elaborating on the spatial explanation of the CPD, some have proposed that filters tuned to high spatial frequencies inhibit responses from filters tuned to low spatial frequencies (Morikawa, 2000; Yeshurun \& Carrasco, 2000; but see Gurnsey et al., 1996). ${ }^{1}$ This hypothesis is based on evidence that sensitivity to high spatial frequencies decreases with eccentricity, whereas sensitivity to low spatial frequencies remains constant across all eccentricities (DeValois \& DeValois, 1988; Graham, Robson, \& Nachmias, 1978; Rovamo, Virsu, \& Näsänen, 1978; Wright \& Johnston, 1983). Thus, interfrequency inhibition may result from the normalization process underlying the two different patterns of frequency sensitivity across eccentricities (Foley, 1994; Graham, Sutter, \& Venkatesan, 1993; Heeger, 1992). In addition to a mismatch between the size of the filter and the scale of the texture, the interfrequency inhibition hypothesis asserts that the CPD results primarily from the participation of nonoptimal high-frequency filters in the normalization process. Given that sensitivity to high-frequency information is highest at central locations, the participation of high-frequency filters at these locations can result in a weakened response of more appropriately scaled lower spatial frequency filters for the task. Indeed, it has been found that removing high spatial frequency information from the texture by low-pass filtering the display virtually eliminates the CPD (Morikawa, 2000, Experiment 5).2

Although many studies have explored the factors that account for the pattern of performance in the texture segmentation task (e.g., Gurnsey et al., 1996; Joffe \& Scialfa, 1995; Kehrer, 1989, 1997; Kehrer \& Meinecke, 2003; von Berg, Ziebell, \& Stiehl, 2002; Yeshurun \& Carrasco, 2000), few studies have investigated the role of attention in shaping this function (Talgar \& Carrasco, 2002; Yeshurun \& Carrasco, 1998, 2000). It has been established that neurons in early visual cortex are capable of carrying out the necessary computations underlying texture segmentation (Lamme, 1995; Purpura, Victor, \& Katz, 1994). Our previous psy- 
chophysical studies on the effects of attention on texture segmentation have established a link between behavioral measures and single-cell (for a recent review, see Reynolds \& Chelazzi, 2004) as well as fMRI (see, e.g., Brefczynski \& DeYoe, 1999; Gandhi, Heeger, \& Boynton, 1999; Liu, Pestilli, \& Carrasco, 2005) studies demonstrating attentional modulation of activity in early visual areas. However, the mechanism by which attention enhances spatial resolution at the level of the primary visual cortex remains unknown.

A possible neural correlate for enhanced spatial resolution is provided by studies showing that attention increases spatial resolution by contracting a neuron's receptive field around the attended stimulus (Luck, Chelazzi, Hillyard, \& Desimone, 1997; Moran \& Desimone, 1985; Reynolds \& Desimone, 1999; Reynolds, Pasternak, \& Desimone, 2000). Alternatively, attention may reduce the filter size by increasing the sensitivity of the smallest receptive fields (Balz \& Hock, 1997) at the attended area, which, via a normalization process, may inhibit the sensitivity of the spatially overlapping larger receptive fields (Yeshurun \& Carrasco, 2000). According to these two alternatives, the maximum sensitivity at the attended regions could shift toward higher spatial frequencies. In a previous study, low-pass filtering the stimulus altered the spatial frequency information in the texture, and the CPD was virtually eliminated (Morikawa, 2000, Experiment 5). Correspondingly, in the present study we use the paradigm of selective adaptation, which allows us to limit the range of spatial frequency filters available to process the texture. In doing so, we can directly investigate not only the mechanisms responsible for the CPD, but also the effect of attention on this texture segmentation task.

If attention increases sensitivity to high spatial frequencies, and if interfrequency inhibition accounts for the CPD, precuing the target location should render the CPD even more pronounced (i.e., CAI). In contrast, weakening the contribution of the high spatial frequencies to the normalization process should diminish or eliminate both the CPD and the CAI. We hypothesized that attention would either improve or impair performance, depending on the availability of spatial frequency information at a given scale of the texture. We directly investigated this hypothesis by precuing the target location while manipulating the spatial frequency information available to the observer via selective adaptation.

\section{The Present Experiment}

We used a method similar to the one used in previous studies investigating the effects of transient attention on texture segmentation tasks (Talgar \& Carrasco, 2002; Yeshurun \& Carrasco, 1998, 2000). We manipulated attention by using an exogenous precue that indicated target location (see, e.g., Carrasco, Giordano, \& McElree, 2004; Carrasco et al., 2000; Golla et al., 2004; Shiu \& Pashler, 1995; Yeshurun \& Carrasco, 1998, 2000).

In addition, to pinpoint the mechanism by which attention enhances spatial resolution, we combined the procedures of cuing and selective adaptation. The selective adaptation procedure is used to assess the spatiotemporal properties of the visual system. It has long been demonstrated that prolonged exposure to one type of stimulus reduces sensitivity to those stimulus parameters and other similar stimuli, thus allowing for the selectivity for a particular variable or set of variables, such as spatial frequency and orientation (Blakemore \& Campbell, 1969; Graham, 1989; Movshon \& Lennie, 1979; Saul \& Cynader, 1989). By manipulating attention during adaptation, researchers have shown that attention modulates the effect of adaptation on the magnitude of motion (e.g., Chaudhuri, 1990), figural (e.g., Suzuki, 2001), and tilt (e.g., Spivey \& Spirn, 2000) aftereffects, and even for illusory contours in the case of tilt aftereffects (Montaser-Kouhsari \& Rajimehr, 2004). In contrast, the present study exploits our knowledge of how selective adaptation alters perceptual input to address the nature of attentional mechanisms and spatial frequency sensitivity across eccentricities. The stimulus content of the texture display that we used remained the same in all experimental conditions. Both attention and spatial frequency content available to the observer were manipulated during the test phase using selective adaptation, which reduces observers' sensitivity to a particular range of frequencies (Blakemore \& Campbell, 1969; Graham, 1989; Movshon \& Lennie, 1979). We utilized selective adaptation as a means to understand how spatial frequency sensitivity affects performance in a texture segmentation task and how attention alters this performance pattern across eccentricities.

This novel paradigm allowed us to characterize the role that high spatial frequencies play in the CPD and to test the hypothesis that attention shifts sensitivity toward higher spatial frequencies, for example:

1. If the CPD was due to the inhibition of lower spatial frequency filters by high spatial frequency filters (which are not optimal for the scale of the texture), adapting to high spatial frequencies should eliminate the CPD (Figure 1A), whereas adapting to low spatial frequencies should have little to no effect on the CPD. Recall that there are relatively fewer low spatial frequency filters in central regions and that their contribution to the normalization process is minimal in central locations in comparison with that of the high spatial frequency filters.

2. If the attentional impairment that was observed at central locations in previous studies (Figure 1B) was due to an increased sensitivity to high frequencies (see, e.g., Yeshurun \& Carrasco, 2000), which in turn reduces sensitivity to lower frequencies, then adapting to high spatial frequencies should eliminate the attentional impairment at central locations and diminish the benefit in the peripheral locations (Figure 1C).

\section{METHOD}

\section{Observers}

Eighteen New York University undergraduate students participated in the study in return for Introduction to Psychology course credit. All had normal or corrected-to-normal vision and were naive as to the purpose of the study. ${ }^{3}$ 
A Selective Adaptation

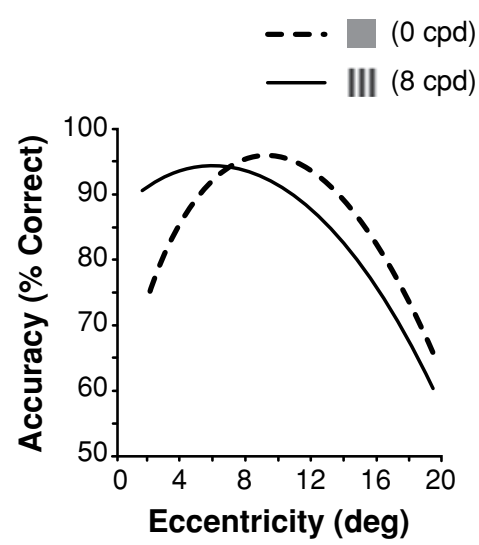

B Attention

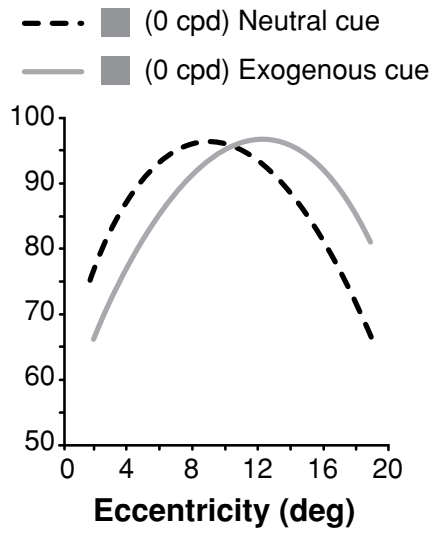

C Selective Adaptation and Attention

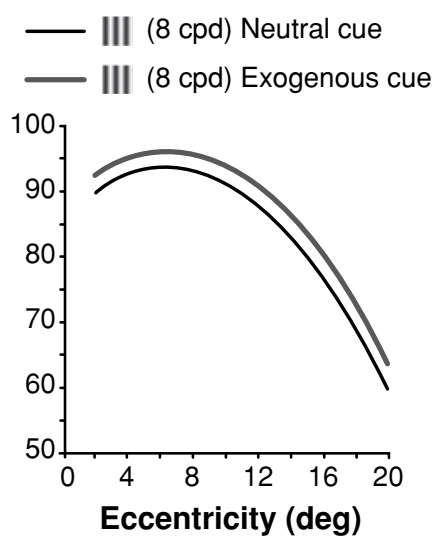

Figure 1. Hypothetical results for the CPD. (A) Selective adaptation. The predicted CPD after adaptation to a high spatial frequency grating of $8 \mathrm{cpd}$ (solid line) compared to a homogeneous gray pattern of $0 \mathrm{cpd}$ - that is, baseline (dotted line). (B) Attention. The CPD with the effect of transient attention using an exogenous cue (solid line) compared with the neutral cue condition (dotted line) after baseline adaptation. (C) Selective adaptation and attention. Predicted results for adaptation to the high spatial frequency grating for the condition in which an exogenous cue (thick line) or neutral cue (thin line) was employed. Note-The predicted curves for the low spatial frequency adaptation condition are not shown in these plots, but we would expect little to no change in the shape of the CPD for this condition; that is, the function should look similar to the standard CPD.

\section{Apparatus}

The stimuli were displayed on a gamma-corrected 21-in. Sony P51 monitor using an Apple G4 computer. The monitor resolution was $1,024 \times 768$ pixels at $75 \mathrm{~Hz}$. A chinrest was used to limit observers' head movements.

\section{Stimuli}

Stimuli were presented using the PsyScope programming software (Cohen, MacWhinney, Flatt, \& Provost, 1993). All texture patterns spanned an area of $5 \times 28 \mathrm{~cm}$ in the center of the monitor screen, with 287 line segments oriented vertically ( 7 rows $\times 41$ columns; $0.3 \mathrm{~cm}$ jitter to avoid grouping). The lines, each subtending $0.2^{\circ} \times$ $1.0^{\circ}$ of visual angle, were presented in black on a white background $(100 \%$ contrast). From a viewing distance of $28 \mathrm{~cm}$, the stimulus texture subtended $10^{\circ} \times 56^{\circ}$ of visual angle. A target patch, composed of $3 \times 3$ lines that were oriented at either $+8^{\circ}$ or $-8^{\circ}$ from vertical and subtending $1.5^{\circ} \times 1.5^{\circ}$ of visual angle, was embedded in a background texture that was composed of vertical lines. The target appeared in 33 different locations across the horizontal meridian, from $0^{\circ}$ to $24^{\circ}$ eccentricity. A homogeneous backward mask consisting of 4 jittered rows of $X$ (whose lines were at $\pm 8^{\circ}$ from vertical) was used to prevent a stimulus afterimage and to avoid ceiling effects (Gurnsey et al., 1996; Joffe \& Scialfa, 1995; Kehrer, 1987, 1989; Meinecke \& Kehrer, 1994); however, it has been shown that a backward mask is not necessary to elicit the CPD (Potechin \& Gurnsey, 2003; Yeshurun \& Carrasco, 2000). To manipulate attention, each display was preceded randomly by either an exogenous or a neutral cue. The exogenous cue, a green horizontal bar subtending $0.6^{\circ} \times 1.2^{\circ}$ of visual angle, appeared $0.6^{\circ}$ above the target location. The neutral cue comprised two horizontal lines, one above and one below the entire texture display.

Three types of stationary adapting patterns were used: homogeneous ( 0 -cpd baseline), high ( 8 -cpd), and low (1-cpd) spatial frequency vertical sinusoidal 100\% contrast gratings (Figure 2). The adapting patterns differed only in their spatial frequency content; thus, any differential effect on performance could be attributed to a change in such content. The orientation of the adapting patterns was vertical in order to adapt both the vertical background texture elements and the slightly tilted target patch, both of which fell in the same bandwidth (see, e.g., Movshon \& Blakemore, 1973; Snowden,
1992). These patterns, subtending $22.5^{\circ} \times 22.5^{\circ}$, were generated in MATLAB (Brainard, 1997; Pelli, 1997). The mean luminance of each pattern was $35 \mathrm{~cd} / \mathrm{m}^{2}$.

\section{Procedure}

After adapting to a high (8-cpd) or low (1-cpd) spatial frequency sinusoidal grating or a homogeneous gray display, the observers' task was to report the orientation (right or left) of the target patch that was embedded in a homogeneous background display of vertical lines (Figure 2) by pressing one of two keys. During the selective adaptation procedure, observers were asked to move their eyes continuously and steadily across the adapting pattern for $4 \mathrm{~min}$. To maintain the effect of adaptation, the same adapting pattern was presented for $4 \mathrm{~min}$ every $3 \mathrm{~min}$ of data collection.

Figure 2 depicts the trial sequence in a two-alternative forced choice orientation discrimination task. Each interval began with a fixation point appearing at the center of the screen for $500 \mathrm{msec}$. An exogenous or a neutral cue was presented for $60 \mathrm{msec}$. After a $60 \mathrm{msec}$ interstimulus interval, the texture was displayed for a duration that ranged from 20 to $60 \mathrm{msec}$ and was adjusted for individual observers to keep overall performance at $80 \%$. The timing between the precue onset and display onset $(120 \mathrm{msec})$ maximized the effect of transient attention (e.g., Carrasco et al., 2004; Cheal \& Lyon, 1991; Nakayama \& Mackeben, 1989; Yeshurun \& Carrasco, 1998) and precluded eye movements (Mayfrank, Kimmig, \& Fischer, 1987).

In half of the trials, an exogenous cue always indicated the target location. In the rest of the trials, a neutral cue indicated that the target had an equal probability of appearing at any location. Both cues provided information about display onset but not about the orientation of the target. The order of the trials for each adaptation condition (cue type, target orientation, target eccentricity) was randomized. After completing a block of 136 practice trials, each observer performed 272 trials per blocked adaptation condition (high, low, and baseline), which were conducted on 3 different days and in a random order.

\section{RESULTS}

Performance was similar in the baseline and lowfrequency conditions but markedly different in the high- 


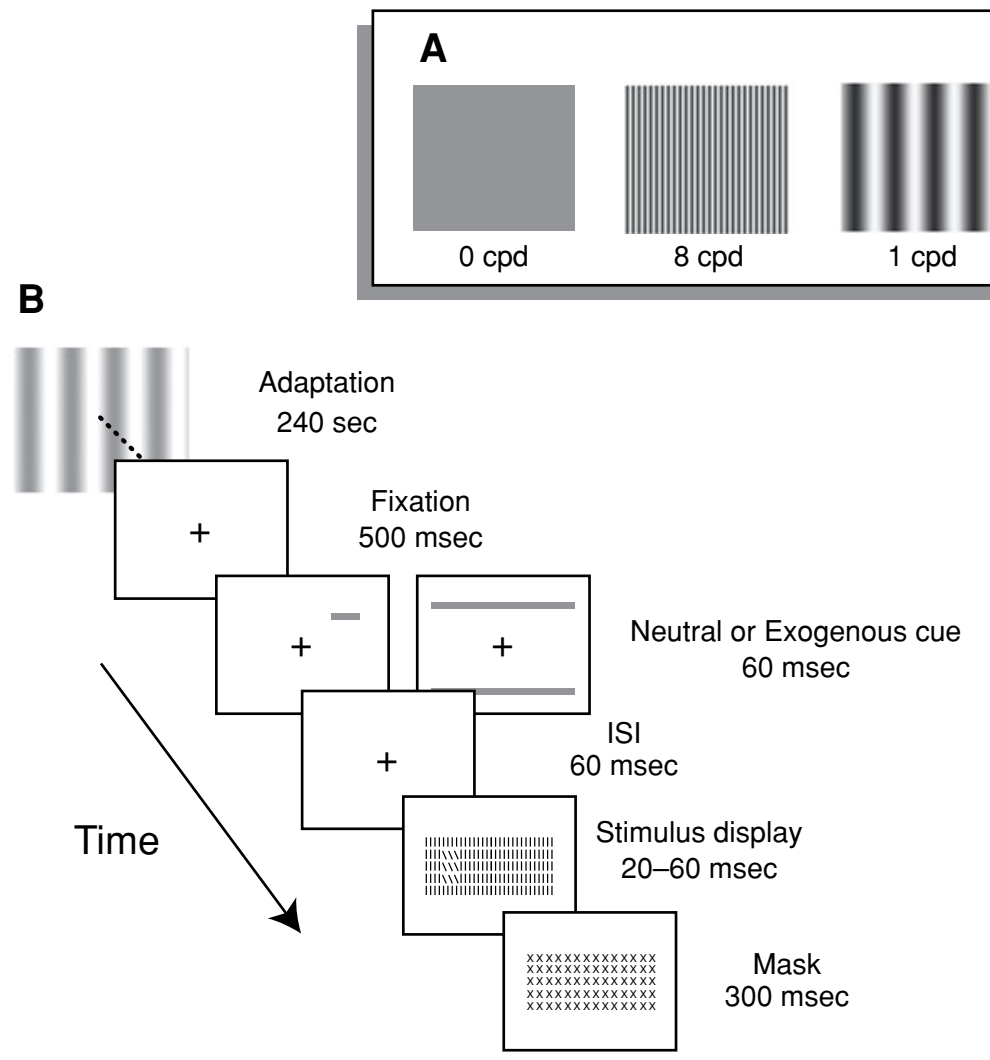

Figure 2. Texture segmentation task. (A) Selective adaptation patterns for three conditions: baseline (left), high spatial frequency (middle), and low spatial frequency (right). (B) Sequence of events in one trial for Experiment 1. Observers performed a two-alternative forced choice discrimination task. (Note-The elements in the texture display were jittered to avoid grouping; jitter not shown here).

frequency condition. Adapting to high but not to low spatial frequencies eliminated both the CPD and CAI. In Figure 3, performance is plotted for both cue types in the three different adaptation conditions. The data were well fitted by second-order polynomials: $r^{2}$ fits ranging from .71 to .94 were obtained (baseline, $r_{\mathrm{exo}}^{2}=.88, r_{\text {neutral }}^{2}=$ .71 ; high, $r_{\mathrm{exo}}^{2}=.85, r_{\text {neutral }}^{2}=.94$; and low, $r_{\mathrm{exo}}^{2}=.87$, $r_{\text {neutral }}^{2}=.92$ ).

A within-observers three-way ANOVA (3 adaptation patterns $\times 2$ cue types $\times 16$ target eccentricities $)^{4}$ revealed main effects of cue type $[F(1,17)=5.39, p<$ $.03]$ and adaptation $[F(2,17)=14.38, p<.001]$. The significant two-way interactions of adaptation $\times$ eccentricity $[F(2,17)=10.37, p<.001]$ and cue type $\times$ eccentricity $[F(1,17)=19.36, p<.001]$ indicated that, as expected, the effect of adaptation and cue type varied as a function of eccentricity. We then analyzed the effects of cue and eccentricity for each of the three adaptation conditions.

The baseline (0-cpd) condition resulted in the typical function found with this texture display: Performance peaked at about $7.3^{\circ}$ eccentricity and decreased at both central and peripheral locations (Figure 3A). This pattern is consistent with previous detection studies (Gurnsey et al., 2004; Gurnsey et al., 1996; Kehrer, 1989;
Morikawa, 2000; Talgar \& Carrasco, 2002; Yeshurun \& Carrasco, 1998, 2000) and extended the occurrence of the CPD to a discrimination task. Cuing the target location affected performance as a function of eccentricity: Accuracy was higher in the exogenous cue trials than in the neutral cue trials at peripheral locations, but lower in the exogenous cue than in the neutral cue trials at central locations - that is, CAI. A significant cue type $\times$ target eccentricity interaction $[F(1,17)=47.3, p<.001]$ confirmed this crossover effect. Furthermore, cuing also displaced the performance peak toward a more peripheral location for each observer (on average, from about $\pm 7.3^{\circ}$ to $\pm 10.3^{\circ}$ eccentricity, Figure $3 \mathrm{~A}$ ). A sign test of the direction of shift in performance peak showed a significant effect of cuing $(p<.001)$. These effects were also consistent with previous texture segmentation studies in which observers in a two-interval forced-choice task were asked to detect whether the target patch appeared in the first or second interval (Talgar \& Carrasco, 2002; Yeshurun \& Carrasco, 1998, 2000).

Consistent with our predictions, adapting to a high spatial frequency grating improved performance in both the neutral and exogenous cue conditions for central locations (Figure 3B). Note that in the neutral cue condition, per- 

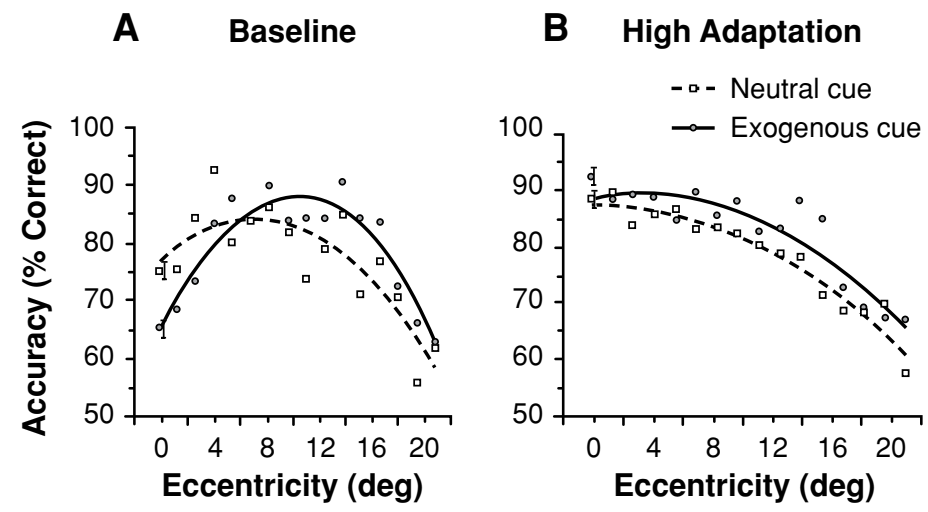

\section{Low Adaptation}

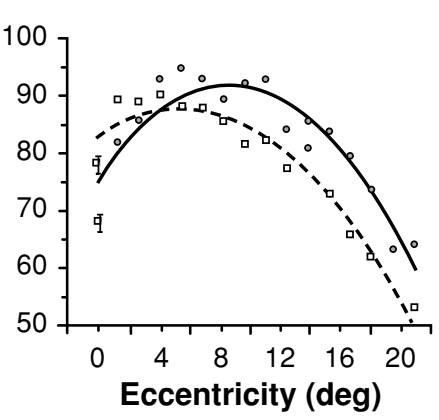

Figure 3. Results. Observers' performance as a function of cue type and target eccentricity. (A) Baseline, (B) high spatial frequency adaptation grating, and (C) low spatial frequency adaptation grating. The error bars correspond to the average standard error for each condition. The data were fitted to second-order polynomials, and $R^{2}$ values ranged from .71 to .94 . Note that the CPD was present in the baseline and the low spatial frequency neutral conditions, but was eliminated in the high spatial frequency neutral condition. Furthermore, the central attentional impairment present in the baseline and low-frequency exogenous cuing conditions was eliminated in the high-frequency exogenous cuing condition.

formance was no longer diminished at central locations. The CPD was eliminated, and performance at central locations decreased continuously with eccentricity. This was in contrast to performance in the baseline condition, which increased by $\sim 10 \%$ from the central locations to the locations where performance peaked. Cuing the target location had a similar effect at all eccentricities - it no longer impaired performance at the central locations. This effect was confirmed by a lack of significant cue type $\times$ target eccentricity interaction $[F(1,17)=1.3, p>.1]$.

Also consistent with our predictions, adapting to a low spatial frequency grating produced a pattern of results similar to that for the baseline condition. The CPD was present in the neutral cue trials and the exogenous cue increased performance at peripheral locations, but the CAI remained (Figure 3C). As in the baseline condition, a significant cue type $\times$ target eccentricity interaction $[F(1,17)=58.7, p<$ $.001]$ confirmed this crossover effect.

\section{DISCUSSION}

We suggest that the CPD is driven primarily by spatial factors, particularly by the contribution of high spatial frequencies, and that the CAI is due to the fact that attention enhances spatial resolution by shifting sensitivity to higher spatial frequencies. We have shown this by implementing a new paradigm that combines cuing with a selective adaptation procedure, which allowed us to effectively alter observers' sensitivity to a selected range of spatial frequencies (Blakemore \& Campbell, 1969; Movshon \& Lennie, 1979). By changing the spatial frequency sensitivity of the visual system, we showed that cuing the target location at different eccentricities can differentially affect performance in a texture segmentation task. These results are consistent with the hypothesis that attention mediates performance by increasing the weight of the smaller scale spatial frequency filters that participate in the normalization process.

\section{How Selective Adaptation Affects the CPD}

Results from this study strongly support the argument for the CPD being driven primarily by spatial factors (see Gurnsey et al., 2004). More specifically, we provide evidence that is consistent with the hypothesis that the CPD is mediated by interfrequency inhibition (Morikawa, 2000; Yeshurun \& Carrasco, 2000). Keeping the stimulus texture content constant in all conditions and employing a selective adaptation procedure enabled us to isolate the contribution of high- and low-frequency content to the performance pattern. At central locations, highfrequency, nonoptimal filters participate in the normalization process, and the weakened response of the optimal filters results in the CPD. Recall that when the texture was low-pass filtered, the CPD was virtually eliminated (Morikawa, 2000). Similarly, in the present study, when observers adapted to high spatial frequencies, the contribution of the nonoptimal filters to the normalization process was minimized and the CPD was diminished. In contrast, when observers adapted to low spatial frequencies, the CPD persisted due to the unaltered response of the nonoptimal frequency filters. This result is consistent with explanations of the CPD as a spatially driven function (see, e.g., Gurnsey et al., 2004; Gurnsey et al., 1996; Kehrer \& Meinecke, 2003; Yeshurun \& Carrasco, 1998, 2000) and provides psychological reality to the argument that the CPD is driven primarily by higher frequency components that are present in the texture.

Interfrequency inhibition has been used to explain a variety of phenomena. One of these is the inability to recognize a spatially undersampled image - for example, Lincoln's portrait (Harmon, 1971). The fact that the face is recognizable when the image of Lincoln is blurred implies that high-frequency information in the image that is due to undersampling may inhibit lower spatial frequency filters from operating. Interfrequency inhibition has also been proposed to explain the grating-induction effect 
(McCourt, 1982), which is characterized by the appearance of a counterphase grating that is produced by placing a homogeneous test field within a grating. The gratinginduction effect decreases when the inducing field contains a grating of high spatial frequency. To explain this finding, McCourt and Foley (1985) proposed a computational model to explain how higher spatial frequency filters may interfere with the processing of optimal low spatial frequency, in a manner consistent with known physiological mechanisms.

Analogous to the effects of selective adaptation on the texture segmentation task reported here, it has been shown that when observers selectively adapt to low - not highspatial frequencies, the magnitude of a length illusion is diminished (Carrasco, Figueroa, \& Willen, 1986). This psychophysical result corresponds to Ginsburg's (1984) finding that in a low - not high - pass filtered image of the Müller-Lyer illusion the inward arrowheads cause the horizontal line segments to be physically shorter in length than the outward arrowheads.

\section{How Selective Adaptation Affects the CAI}

Attention affects performance in resolution tasks such as visual search (Cameron, Tai, Eckstein, \& Carrasco, 2004; Carrasco \& Yeshurun, 1998), acuity and hyperacuity (Carrasco et al., 2002; Golla et al., 2004; Shiu \& Pashler, 1995; Yeshurun \& Carrasco, 1999), and texture segmentation (Talgar \& Carrasco, 2002; Yeshurun \& Carrasco, 1998, 2000). The hypothesis that attention enhances spatial resolution has inspired neuronal models that implement the role that visual attention plays in object recognition (see Deco, Rolls, \& Zihl, 2005) and has also been captured in a computational model proposing that interactions among visual spatial filters result in both increased gain and sharpened tuning (see, e.g., Lee et al., 1999).

In the present study, we investigated whether attention influences the normalization process by increasing sensitivity to higher spatial frequencies. Consistent with this hypothesis and our previous findings (Talgar \& Carrasco, 2002; Yeshurun \& Carrasco, 1998, 2000), we found that precuing the target location rendered the CPD even more pronounced in the baseline condition. Moreover, when, via selective adaptation, the contribution of the nonoptimal high frequencies was diminished in the normalization process, precuing the target location no longer impaired performance, as if optimal lower frequency filters were no longer inhibited. Indeed, we observed no attentional impairment at central locations after adaptation to high spatial frequencies. In contrast, after adaptation to low spatial frequencies, the central attentional impairment was still present, due to the unaltered input from nonoptimal high spatial frequency filters in the normalization process.

These results confirm that attention affects early stages of visual processing, and, more importantly, they provide strong support for our hypothesis that attention enhances spatial resolution by shifting sensitivity to higher spatial frequencies. This attentional mechanism can ac- count for the present results as well as for our previous results (Talgar \& Carrasco, 2002; Yeshurun \& Carrasco, 1998, 2000). Furthermore, the mechanism put forth by this study provides a basis for two findings from a recent study showing that transient attention can (1) alter the perceived spatial frequency of a stimulus in such a way that the spatial frequency of a cued target appears higher than it actually is, and (2) increase the perceived gap size of a Landolt square, making the gap more resolvable (Gobell \& Carrasco, 2005).

Could an alternative mechanism explain these results? We know, for instance, that attention affects performance not only by affecting discriminability but also by altering the speed with which information is processed. Specifically, given that transient attention increases speed of processing (Carrasco, Giordano, \& McElree, 2004, 2006; Carrasco \& McElree, 2001), we would expect attention to improve performance at all target locations, regardless of the adaptation condition. This, however, was not the case: Attention impaired performance at the central locations in the baseline and the low-frequency adaptation conditions and improved it at peripheral locations in all three adaptation conditions.

Some might also argue that attention effects can be due to reduction of uncertainty at the cued location (see, e.g., Eckstein, 1998; Foley \& Schwartz, 1998; Palmer, Verghese, \& Pavel, 2000). According to this model, attention should improve performance at all target locations. Again, however, attention impaired performance at the central locations in the baseline and in the low-frequency adaptation conditions. Moreover, given that target/background discriminability decreases and location uncertainty increases with eccentricity, one would expect that the magnitude of the attentional benefit would increase with eccentricity. This, however, was not the case: Attention improved performance to a similar degree at peripheral locations in all three adaptation conditions.

\section{CONCLUSION}

By diminishing the availability of high spatial frequency information, we eliminated the CPD. This study provides evidence supporting the interfrequency inhibition explanation of the CPD. The spatial interpretation of the CPD has been used to effectively model data from several experiments (von Berg et al., 2002), and our present finding could also account for the findings of those studies.

Additionally, by using selective adaptation in conjunction with a cuing paradigm, we eliminated the CAI by diminishing the availability of high spatial frequency information. This finding indicates that attention enhances spatial resolution by shifting sensitivity to higher spatial frequencies in texture segmentation tasks. This mechanism can account for the effects of attention in visual search (see, e.g., Cameron et al., 2004; Carrasco \& Yeshurun, 1998; Nakayama \& Mackeben, 1989), acuity and hyperacuity (see, e.g., Carrasco et al., 2002; Golla et al., 
2004), and orientation discrimination (see, e.g., Baldassi \& Burr, 2000; Carrasco, Talgar, \& Cameron, 2001; Morgan et al., 1998) tasks.

\section{REFERENCES}

BALDASSI, S., \& BURR, D. C. (2000). Feature-based integration of orientation signals in visual search. Vision Research, 40, 1293-1300.

BALz, G. W., \& HocK, H. S. (1997). The effect of attentional spread on spatial resolution. Vision Research, 37, 1499-1510.

Blakemore, C., \& Campbell, F. W. (1969). On the existence of neurons in the human visual system selectively sensitive to the orientation and size of retinal images. Vision Research, 203, 237-260.

Brainard, D. J. (1997). The Psychophysics Toolbox. Spatial Vision, 10, 433-436.

BREFCZYNSKI, J. A., \& DeYoe, E. A. (1999). A physiological correlate of the 'spotlight' of visual attention. Nature Neuroscience, 2, 370-374.

BReITMEYer, B. (1984). Visual masking: An integrative approach. New York: Oxford University Press.

Cameron, E. L., Tai, J. C., \& Carrasco, M. (2002). Covert attention affects the psychometric function of contrast sensitivity. Vision Research, 42, 949-967.

Cameron, E. L., Tai, J. C., Eckstein, M. P., \& Carrasco, M. (2004). Signal detection theory applied to three visual search tasks: Identification, yes/no detection and localization. Spatial Vision, 17, 295-325.

Carrasco, M., Figueroa, J. G., \& Willen, J. D. (1986). A test of the spatial-frequency explanation of the Müller-Lyer illusion. Perception, $15,553-562$

Carrasco, M., \& Frieder, K. S. (1997). Cortical magnification neutralizes the eccentricity effect in visual search. Vision Research, $\mathbf{3 7}$ 63-82.

Carrasco, M., Giordano, A. M., \& McElree, B. (2004). Temporal performance fields: Visual and attentional factors. Vision Research, 44, 1351-1365.

Carrasco, M., Giordano, A. M., \& McElree, B. (2006). Attention speeds processing across eccentricity: Feature and conjunction searches. Vision Research, 46, 2028-2040.

Carrasco, M., \& McElree, B. (2001). Covert attention accelerates the rate of visual information processing. Proceedings of the National Academy of Sciences, 98, 5363-5367.

Carrasco, M., McElree, B., Denisova, K., \& Giordano, A. M. (2003). Speed of visual processing increases with eccentricity. Nature Neuroscience, 6, 669-670.

Carrasco, M., Penpeci-Talgar, C., \& Eckstein, M. (2000). Spatial covert attention increases contrast sensitivity along the CSF: Support for signal enhancement. Vision Research, 40, 1203-1215.

Carrasco, M., Talgar, C., \& Cameron, L. (2001). Characterizing visual performance fields: Effects of transient covert attention, spatial frequency, eccentricity, task, and set size. Spatial Vision, 14, 61-75.

Carrasco, M., Williams, P., \& Yeshurun, Y. (2002). Covert attention increases spatial resolution with or without masks: Support for signal enhancement. Journal of Vision, 2, 467-479. Available at journalofvision.org/2/6/4/, doi:10.1167/2.6.4.

Carrasco, M., \& Yeshurun, Y. (1998). The contribution of covert attention to the set-size and eccentricity effects in visual search. Journal of Experimental Psychology: Human Perception \& Performance, 24, 673-692.

Chaudhuri, A. (1990). Modulation of the motion aftereffect by selective attention. Nature, 344, 60-62

Cheal, M. L., \& Lyon, D. R. (1991). Central and peripheral precuing of forced-choice discrimination. Quarterly Journal of Experimental Psychology, 43A, 859-880.

Cohen, J. D., MacWhinney, B., Flatt, M., \& Provost, J. (1993). PsyScope: A new graphic interactive environment for designing psychology experiments. Behavior Research Methods, Instruments, \& Computers, 25, 257-271.

Deco, G., Rolls, E. T., \& Zinl, J. (2005). A neurodynamical model of visual attention. In L. Itti, G. Rees, \& J. K. Tsotsos (Eds.), Neurobiology of attention (pp. 593-599). Amsterdam: Elsevier.
DeValois, R. L., Albrecht, D. G., \& Thorell, L. G. (1982). Spatial frequency selectivity of cells in macaque visual cortex. Vision Research, 22, 545-559.

DeValois, R. L., \& DeValois, K. K. (1988). Spatial vision. New York: Oxford University Press.

Dosher, B., \& LU, Z.-L. (2000a). Mechanisms of perceptual attention in precuing of location. Vision Research, 40, 1269-1292.

Dosher, B., \& Lu, Z.-L. (2000b). Noise exclusion in spatial attention. Psychological Science, 11, 139-146.

EcKstein, M. P. (1998). The lower efficiency for conjunctions is due to noise and not serial attentional processing. Psychological Science, 9, 111-118.

Foley, J. M. (1994). Human luminance pattern-vision mechanisms: Masking experiments require a new model. Journal of the Optical Society of America A, 11, 1710-1719.

Foley, J. M., \& Schwartz, W. (1998). Spatial attention: Effect of position uncertainty and number of distractor patterns on the threshold-versus-contrast function for contrast discrimination. Journal of the Optical Society of America, 15, 1036-1047.

Gandhi, S. P., Heeger, D. J., \& Boynton, G. M. (1999). Spatial attention affects brain activity in human primary visual cortex. Proceedings of the National Academy of Sciences, 96, 3314-3319.

GiNSBURG, A. P. (1984). Visual form perception based on biological filtering. In L. Spilman \& U. R. Woolen (Eds.), Sensory experiences, adaptation, and perception (pp. 53-72). Hillsdale, NJ: Erlbaum.

Gobell, J., \& CARRASCO, M. (2005). Attention alters the appearance of spatial frequency and gap size. Psychological Science, 16, 644-651.

Golla, H., Ignashchenkova, A., HaArmeier, T., \& Their, P. (2004). Improvement of visual acuity by spatial cueing: A comparative study in human and non-human primates. Vision Research, 44, 1589-1600.

Graham, N. (1989). Visual pattern analyzers. New York: Oxford University Press.

Graham, N., Robson, J. G., \& Nachmias, J. (1978). Grating summation in fovea and periphery. Vision Research, 18, 815-825.

Graham, N., \& SutTer, A. (2000). Normalization: Contrast-gain control in simple (Fourier) and complex (non-Fourier) pathways of pattern vision. Vision Research, 40, 2737-2761.

Graham, N., Sutter, A., \& Venkatesan, C. (1993). Spatial-frequency and orientation-selectivity of simple and complex channels in region segregation. Vision Research, 33, 1893-1911.

Gurnsey, R., Di Lenardo, D., \& Potechin, C. (2004). Backward masking and the central performance drop. Vision Research, 44, 2587-2596.

Gurnsey, R., Pearson, P., \& Day, D. (1996). Texture segmentation along the horizontal meridian: Nonmonotonic changes in performance with eccentricity. Journal of Experimental Psychology: Human Perception \& Performance, 22, 738-757.

HARMON, L. (1971). Some aspects of recognition of human faces. In O. J. Grusser (Ed.), Pattern recognition in biological and technical systems. Berlin: Springer.

HeEger, D. J. (1992). Normalization of cell responses in cat striate cortex. Visual Neuroscience, 9, 181-198.

Joffe, K. M., \& Scialfa, C. T. (1995). Texture segmentation as a function of eccentricity, spatial frequency and target size. Spatial Vision, 9, 325-342.

JoNIDES, J. (1981). Voluntary vs. automatic control over the mind's eye's movement. In J. B. Long \& A. D. Baddeley (Eds.), Attention and performance IX (pp. 187-204). Hillsdale, NJ: Erlbaum.

Kehrer, L. (1987). Perceptual segregation and retinal position. Spatial Vision, 2, 247-261.

KeHrer, L. (1989). Central performance drop on perceptual segregation tasks. Spatial Vision, 4, 45-62.

KeHrer, L. (1997). The central performance drop in texture segmentation: A simulation based on a spatial filter model. Biological Cybernetics, 77, 297-305.

Kehrer, L., \& Meinecke, C. (2003). A space-variant filter model of textures segregation: Parameter adjustment guided by psychophysical data. Biological Cybernetics, 88, 183-200.

Lamme, V. A. F. (1995). The neurophysiology of figure-ground segregation in primary visual cortex. Journal of Neuroscience, 15, 1605-1615. 
Lee, D. K., Itti, L., Koch, C., \& Braun, J. (1999). Attention activates winner-take-all competition among visual filters. Nature Neuroscience, 2, 375-381.

Ling, S., \& CARrasco, M. (2006). Sustained and transient covert attention enhance the signal via different contrast response functions. Vision Research, 46, 1210-1220.

Liu, T., Pestilli, F., \& Carrasco, M. (2005). Transient attention enhances perceptual performance and $\mathrm{fMRI}$ response in human visual cortex. Neuron, 45, 469-477.

Lu, Z.-L., \& Dosher, B. A. (1998). External noise distinguishes attention mechanisms. Vision Research, 38, 1183-1198.

Lu, Z.-L., \& Dosher, B. A. (2000). Spatial attention: Different mechanisms for central and peripheral temporal precues? Journal of Experimental Psychology: Human Perception \& Performance, 26, 1534-1548.

Luck, S. J., Chelazzi, L., Hillyard, S. A., \& Desimone, R. (1997). Neural mechanisms of spatial selective attention in areas V1, V2, and V4 of macaque visual cortex. Journal of Neurophysiology, 77, 24-42.

Mayfrank, L., Kimmig, H., \& Fischer, B. (1987). The role of attention in the preparation of visually guided saccadic eye movements in man. In J. K. O'Regan \& A. Levy-Schoen (Eds.), Eye movements: From physiology to cognition (pp. 37-45). New York: Elsevier, NorthHolland.

McCourt, M. E. (1982). A spatial frequency dependent grating induction effect. Vision Research, 22, 119-134.

McCourt, M. E., \& Foley, J. M. (1985). Spatial frequency interference on grating-induction. Vision Research, 25, 1507-1518.

Meinecke, C., \& Kehrer, L. (1994). Peripheral and foveal segmentation of angle textures. Perception \& Psychophysics, 56, 326-334.

Montaser-Kouhsari, L., \& Rajimehr, R. (2004). Attentional modulation of adaptation to illusory lines. Journal of Vision, 4, 434-444. Available at journalofvision.org/4/6/3/, doi:10.1167/4.6.3.

Moran, J., \& Desimone, R. (1985). Selective attention gates visual processing in the extrastriate cortex. Science, 229, 782-784.

Morgan, M. J., WARD, R. M., \& CASTET, E. (1998). Visual search for a tilted target: Tests of spatial uncertainty models. Quarterly Journal of Experimental Psychology, 51A, 343-370.

MoriKawA, K. (2000). Central performance drop in texture segmentation: The role of spatial and temporal factors. Vision Research, $\mathbf{4 0}$ 3517-3526.

Movshon, J. A., \& Blakemore, C. (1973). Orientation specificity and spatial selectivity in human vision. Perception, 2, 53-60.

Movshon, J. A., \& Lennie, P. (1979). Pattern selective adaptation in visual cortical neurons. Nature, 278, 850-852.

Nakayama, K., \& Mackeben, M. (1989). Sustained and transient components of focal visual attention. Vision Research, 29, 1631-1646.

Palmer, J., Verghese, P., \& Pavel, M. (2000). The psychophysics of visual search. Vision Research, 40, 1227-1268.

Pelli, D. G. (1997). The VideoToolbox software for visual psychophysics: Transforming numbers into movies. Spatial Vision, 10, 437-442.

Posner, M. I. (1980). Orienting of attention. Quarterly Journal of Experimental Psychology, 32, 3-25.

Potechin, C., \& GuRnsey, R. (2003). Backward masking is not required to elicit the central performance drop. Spatial Vision, 16, 393-406.

Purpura, K. P., Victor, J. D., \& Katz, E. (1994). Striate cortex extracts higher-order spatial correlations from visual textures. Proceedings of the National Academy of Sciences, 91, 8482-8486.

Reynolds, J. H., \& ChelazzI, L. (2004). Attentional modulation of visual processing. Annual Review of Neuroscience, 27, 611-647.
Reynolds, J. H., \& Desimone, R. (1999). The role of neural mechanisms of attention in solving the binding problem. Neuron, 24, 19-29.

Reynolds, J. H., Pasternak, T., \& Desimone, R. (2000). Attention increases sensitivity of V4 neurons. Neuron, 26, 703-714.

Rovamo, J., Virsu, V., \& NÄsÄnen, R. (1978). Cortical magnification factor predicts the photopic contrast sensitivity of peripheral vision. Nature, 271, 54-56.

Saul, A. B., \& Cynader, M. S. (1989). Adaptation in single units in visual cortex: The tuning of aftereffects in the spatial domain. Visual Neuroscience, 2, 593-607.

Shiu, L., \& Pashler, H. (1995). Spatial attention and vernier acuity. Vision Research, 35, 337-343.

SNOWDEN, R. (1992). Orientation bandwidth: The effect of spatial and temporal frequency. Vision Research, 32, 1965-1974.

SPIVEY, M. J., \& SPIRN, M. J. (2000). Selective visual attention modulates the direct tilt aftereffect. Perception \& Psychophysics, 62, 1525-1533.

SuzUKI, S. (2001). Attention-dependent brief adaptation to contour orientation: A high-level aftereffect for convexity? Vision Research, $\mathbf{4 1}$ 3883-3902.

TAlgar, C. P., \& CARrasco, M. (2002). Vertical meridian asymmetry in spatial resolution: Visual and attentional factors. Psychonomic Bulletin \& Review, 9, 714-722.

TSAL, Y., \& SHALEV, L. (1996). Inattention magnifies perceived length: The attentional receptive field hypothesis. Journal of Experimental Psychology: Human Perception \& Performance, 22, 233-243.

von Berg, J., Ziebell, O., \& Stiehl, H. S. (2002). Texture segmentation performance related to cortical geometry. Vision Research, 42, 1917-1929.

Wright, M. J., \& Johnston, A. (1983). Spatiotemporal contrast sensitivity and visual field locus. Vision Research, 23, 983-989.

Yeshurun, Y., \& CARRASCO, M. (1998). Attention improves or impairs visual performance by enhancing spatial resolution. Nature, 396, 72-75.

Yeshurun, Y., \& CARRASCO, M. (1999). Spatial attention improves performance in spatial resolution tasks. Vision Research, 39, 293-305.

Yeshurun, Y., \& CARRASCO, M. (2000). The locus of attentional effects in texture segmentation. Nature Neuroscience, 3, 622-627.

\section{NOTES}

1. Gurnsey et al. (1996) filtered out high spatial frequencies from the stimulus texture and mask. They did not support the interfrequency inhibition hypothesis because performance at the fovea did not increase as more high frequencies were filtered out. However, notice that their Figure 7 ( $p$. 748 ) illustrates that the magnitude of the CPD - that is, the difference between the level of performance at central locations and at the peak level decreased significantly and in an orderly fashion with increased blurring.

2. Some of the conclusions reached by Morikawa in Experiments 2 and 3 have been criticized (Potechin \& Gurnsey, 2003), but the finding of Experiment 5 has not been contested.

3. The New York University Committee on Activities Involving Human Subjects, Office of Sponsored Programs, approved the experimental protocol

4. Data were collapsed across the right and left visual fields because there were no significant differences across hemifields $(p>.1)$.

(Manuscript received June 27, 2005; revision accepted for publication October 12, 2005.) 